\title{
Myocardial conditioning techniques in off-pump coronary artery bypass grafting
}

\author{
Marco Moscarelli ${ }^{1,2^{*}}$, Prakash P Punjabi ${ }^{1}$, Gamov I Miroslav ${ }^{1}$, Paolo Del Sarto ${ }^{2}$, Francesca Fiorentino ${ }^{1}$ \\ and Gianni D Angelini $i^{1,3}$
}

\begin{abstract}
Off-pump coronary artery bypass surgery by avoiding cardioplegic arrest seems to reduce the risk of ischemic myocardial injury. However, even short-term regional ischemic periods, hemodynamic instability and arrhythmias associated with the procedure can be responsible for myocardial damage. Conditioning, a potential cardio-protective tool during on-pump cardiac surgery, has hardly been investigated in the context of off-pump surgery. There are virtually no large trials on remote ischemic preconditioning and the majority of reports have focused on central ischemic conditioning. Similarly, volatile anesthetic agents with conditioning effect like ischemic preconditioning have been shown to reduce cardiac injury during on-pump procedures but have not been validated in the off-pump scenario. Here, we review the available evidence on myocardial conditioning, either with ischemia/reperfusion or volatile anesthetic agents in patients undergoing off-pump coronary artery surgery.
\end{abstract}

Keywords: Off-pump coronary artery bypass, Ischemic preconditioning, Volatile anaesthetics

\section{Review}

Conditioning is an umbrella definition that consists of pre-conditioning, per-conditioning and post-conditioning [1]. Conditioning can be elicited remotely (e.g. at the level of a limb) [2] or centrally (e.g. at the level of the heart) [3]. Stimulus normally consists of an ischemic period followed by reperfusion [4], but other triggers like pain-stimulus [5], hyperbaric oxygen [6] and most importantly methods such as volatile anesthetics [7] have been advocated as being equally effective. Conditioning has been experimented in cardiac surgery mainly in the form of remote ischemic preconditioning (RIPC) and the majority of the largest trials have been in on-pump CABG patients (ONCAB) [8] or during valve surgery [9]. RIPC seems to lowers troponin release in patients undergoing ONCAB ('proof of concept'), but still unclear is if this leads to any better clinical outcome. RIP Heart-Study will recruit over 2000 on-pump patients and will include as primary outcomes all-cause mortality, non-fatal MI, any new stroke and/or acute renal failure [10] and the ERICCA [11] trial will establish if there would be better clinical outcome in on-pump high-

\footnotetext{
* Correspondence: m.moscarelli@imperial.ac.uk

${ }^{1} \mathrm{NHLI}$, Hammersmith Hospital, Imperial College London, London, UK

${ }^{2}$ Fondazione Monasterio, Ospedale Pasquinucci, Massa, Italy

Full list of author information is available at the end of the article
}

risk patients. However, it is still an open question whether conditioning has potential benefits in patients undergoing $\mathrm{OPCAB}$ in terms of lowering troponin release and improving clinical outcomes. Ischemia during OPCAB can happen in $10 \%$ of cases [12] with ST segment elevation in up to $40 \%$ of patients [13]. Although the use of intracoronary shunts can reduce the ischemic time, even shortterm regional ischemic periods or heart manipulations can result in myocardial injury [14], and subsequent arrhythmias and/or hemodynamic instability which can lead even to conversion to ONCAB [15].

Several reports on the use of volatile anesthetics cardio-protective agents in ONCAB surgery have suggested a similar ischemic preconditioning effect. However, only small trials have investigated the effect of anaesthetic preconditioning in OPCAB. The use of alternative methods such as adenosine, hyperbaric oxygen or temperature in OPCAB is also yet to be validated. Optimizing protection of the heart from ischemia/reperfusion injury (I/R) during $\mathrm{OPCAB}$ is, therefore, a worthwhile goal.

\section{Ischemic conditioning in OPCAB}

Although there are evidences supporting the benefits of ischemic preconditioning (IP) in terms of cardio-protection, 
its adoption in OPCAB has been relatively limited (Table 1 ) with just few studies published so far.

Malkowsky et al. [13] induced IP with 5 minutes of local coronary artery occlusion and 5 minutes of reperfusion in a series of 17 single vessel OPCAB patients. Left anterior descending artery (LAD) occlusion/reperfusion increased left ventricle wall motion score (LVWMS) and pulmonary pressure (PA). However, subsequent ischemia during the construction of the anastomosis did not alter the regional LV systolic function. van Aarnhem et al. [12] retrospectively reviewed a cohort of 200 OPCAB patients in whom ischemic preconditioning was used before anastomosis by occluding the vessel for $5 \mathrm{mi}$ nutes and then allowing 5 minutes of reperfusion. They reported a $10 \%$ incidence of-intra operative ischemia (defined as $1 \mathrm{~mm}$ ST segment elevation) but no perioperative myocardial injury. Intra-coronary shunts were used in case of critical ischemia and no conversion to ONCAB was observed. Laurikka et al. [14] showed that IP, induced with two cycles of 2 minutes left anterior descending artery occlusion (LAD) followed by 3 minutes of reperfusion before the first coronary artery anastomosis, decreased the immediate myocardial enzyme release, reduced the post-operative increase in $H R$, and enhanced the recovery of volume index after surgery. Doy et al. demonstrated that a single cycle of 5 minutes of central I/R attenuated ischemia-induced electrophysiological changes in patients undergoing MIDCAB [23]. Wu et al. [22] reported that IP, induced by to cycles of 2 minutes occlusion of LAD followed by reperfusion, led to a positive suppression of HR and reduced the incidence of supra-ventricular and ventricular arrhythmias, although the incidence of post-operative atrial fibrillation (AF) remained similar between preconditioned and non-preconditioned groups. Drengen et al. [21] compared prospectively a cohort of patients preconditioned with a single cycle of 5 minutes of LAD occlusion followed by 5 minutes of reperfusion and a cohort preconditioned with $1.6 \%$ enflurane with a non-preconditioned group. They reported a significant reduction of metabolic deficit in both the IP and volatile groups compared to the non-preconditioned group [21]. Hong et al. [19] carried out a prospective controlled randomized trial on RIPC in patients undergoing OPCAB. Remote ischemic preconditioning was elicited with 4 cycles of 5 min upper limb ischemia and 5 min of reperfusion after anesthesia. Troponin release was lower in the RIPC group but did not reach statistical significance $(p=0.172)$ [19]. Succi et al. [20] reported that IP, induced only by 1 minutes of LAD occlusion followed by 2 minutes of reperfusion before anastomosis prevented decrease in left ventricular contractility Hong et al. [18] used both RIPC and postconditioning (PostC) in a cohort of 35 OPCAB patients. Ischemic remote preconditioning and PostC were elicited with 4 cycles of 5 minutes of ischemia and 5 minutes of reperfusion of the lower limb before and after anastomosis. They reported a significant decrease in post-operative myocardial enzymes. Forouzinna et al. [17] prospectively compared patients preconditioned either with adenosine or with $2 \mathrm{cy}-$ cles of 2 minutes of LAD occlusion followed by reperfusion with a non preconditioned control group. They reported no differences in terms of post-operative EF and enzyme release but the incidence of post-operative AF was higher in the IP group although it did not reach statistical significance. A recent pilot study by Jung et al. focusing on neurological outcome did not show any benefits of RIPC in patients undergoing $\mathrm{OPCAB}$ in terms of cognitive outcomes [16].

\section{Volatile anesthetic agents conditioning in OPCAB}

Several reports investigating the potential use of volatile anesthetics as preconditioning agents in OPCAB (Table 2) have reported rather different results. Sevoflurane was found to reduce troponin release in three trials [25-27], although Orriach et al. used sevoflurane as well as postconditioning agent, extending its administration during the first post-operative hours [25]. No differences in troponin release were reported by other authors when sevoflurane was compared to desflurane and propofol [28], to propofol in a remifentanil-based anesthesia regime $[29,30]$ and to isoflurane alone [31,32]. Sevoflurane was also showed to have several other benefits: better antioxidative properties than propofol [33], reduced incidence of arrhythmias compared to desflurane [34], better preservation of cardiac function compared to control and to propofol $[35,36]$ and to reduced NBP release and plasma protein A release when compared to propofol [37]. Isoflurane was reported to reduce troponin release when compared to propofol [38] but no differences were found when compared to sevoflurane $[31,32]$. Other authors on the contrary did not find differences in terms of troponin release when it was compared to a propofol group of patients [39]. Desflurane was reported to improve LSWI when compared to a propofol anesthetic regime, although no differences in troponin release were observed [40], and moreover was found to be less effective in reducing incidence of arrhythmias than sevoflurane [34]. Guarracino et al. observed a significant reduction in troponin release in a cohort of 57 patients anesthetized with desflurane [41]. Finally, remifentanil was found to have a preconditioning effect by lowering troponin release [42], however no differences were reported when was associated with sevoflurane and compared to propofol [30].

\section{Alternative way to standard conditioning in OPCAB}

There have been few reports on the use of adenosine as preconditioning agent in OPCAB. Forouzinna et al. [17] reported no differences between preconditioned and non 
Table 1 Studies including ischemic conditioning

\section{Author, date, journal and} country study type

Joung et al. (2013) Korean

J Anestesiol, Korea [16]

Prospective controlled

randomized trial

Forouzannia et al. (2013)

J The Univ Heart Ctr, Iran

[17] Prospective controlled

randomized tria

Hong et al. (2012) Circulation Journal, Japan [18] Prospective controlled randomized trial

Hong et al. (2010)] Anaesth Intensive Care, Korea [19]

Prospective randomized

controlled trial

Succi et al. (2010) Arq Bras Cardiol, Brasil [20] Prospective controlled randomized trial

Drenger et al. (2008) Journal of Cardiothoracic and Vascular Anesthesia, Israel [21] Prospective controlled randomized trial

Wu et al. (2003) Journal of Cardiothoracic and Vascular Anesthesia, Finland [22]

Prospective controlled randomized trial

\section{Patient group Type of conditioning}

Seventy OPCAB

35 RIPC

35 Control

Sixty OPCAB

20 Adenosine

$20 \mathrm{IP}$

20 Control

Seventy OPCAB

$35 \mathrm{RIPC}+$

RIPpostC

35 Control

130 OPCAB

65 RIPC

65 Control

Forty OPCAB

$0 \mathrm{IP}$

20 Control

Twenty five

OPCAB

8 Control

9 IP

8 Enflurane

Thirty two

OPCAB

16 IP

16 Control
RIPC 4 cycles of 5 min ischemia and $5 \mathrm{~min}$ of reperfusion before coronary artery anastomoses

Adenosine

IP induced with twice $2 \mathrm{~min}$

LAD occlusion followed $3 \mathrm{~min}$ reperfusion before the first anastomosis

Lower limb 4 cycles of 5 min ischemia and $5 \mathrm{~min}$ of reperfusion before anastomoses (RIPC) and after anastomoses (RIPostC)

pper limb 4 cycles of 5 min ischemia and 5 min of

reperfusion after anesthesia

$P$ induced with twice $1 \mathrm{~min}$ AD occlusion followed $2 \mathrm{~min}$ reperfusion before the anastomosis

$\mathrm{P}$ induced with single $5 \mathrm{~min}$ LAD occlusion followed by 5 min reperfusion $1.6 \%$ ENF started 15 min before LAD occlusion

IP induced with twice $2 \mathrm{~min}$ LAD occlusion followed $3 \mathrm{~min}$ reperfusion before the first anastomosis

\section{Key results}

Post-operative cognitive dysfunction

Six cognitive

function test day 1 was 28.6\% (10 pts) and 31.4\% (11 pts) after surgery

in RIPC and Control group respectively

Post-op EF

Arrhythmias

Troponin release

Troponin release

Intra-op EF

(measured pulsed

Doppler of the

descending

thoracic aorta)

Myocardial

metabolism

Incidence of post-operative

arrhythmias postoperative serum troponin I levels

Troponin release was lower in the RIPC group but was not statistically significant

IP and adenosine did not elicit compared to the control group

celeration of the aortic blood flow with no differences in between groups; IP group maintained left ventricular contractility during the entire procedure while the contro group presented significant reduction in left ventricular contractility

Lactate production in the ENF group decreased significantly compared with control and IP groups. Oxygen utilization in the control was $44 \%$ higher than the other two groups. Early recovery of anterior wall hypokinesis in both

study group

IP suppressed the HR elevation during the time of myocardial ischemia and reperfusion and significantly reduced the incidence of $\mathrm{VT}$ after surgery. Incidence of SVT during 2 to 24 hours after surgery was lower in the IP patients but incidence of SVES, VES, and AF were similar between the 2 groups statistically significant EF preservation
Comments

PC did not reduce incidence of

ost-op cognitive dysfunction

after OPCABG during the immediate post-op period

No difference found in post-op E and enzymes release in between groups. Incidence of arrhythmias was higher in the IP group but did not reach statistical significance

$\mathrm{RIPC}+\mathrm{RIPostC}$ decreased postoperative myocardial enzyme elevation by almost half postoperatively in patients undergoing OPCAB

RIPC did not reduce significantly post-operative myocardial

enzyme release

prevented the decrease in left ventricular contractility during

off-pump myocardial

revascularization surgery

Application of methods such as IP or volatile anesthesia appeared to reduce the metabolic deficit

Arrhythmia was a common phenomenon during and after OPCAB procedure; IP protocol significantly suppressed HR elevation, episodes of SVT, and incidence of $\mathrm{VT}$ after surgery but incidence of post-op AF was similar in between groups 
Table 1 Studies including ischemic conditioning (Continued)

\begin{tabular}{|c|c|c|c|c|c|}
\hline $\begin{array}{l}\text { Doi et al. (2003) Jpn J Thorac } \\
\text { Cardiovasc Surg, Japan [23] } \\
\text { Prospective observational study }\end{array}$ & $\begin{array}{l}\text { Forty-five } \\
\text { OPCAB } \\
\text { (MIDCAB) }\end{array}$ & $\begin{array}{l}\text { IP induced with } 5 \text { min vessel } \\
\text { occlusion followed } 5 \text { min } \\
\text { reperfusion before anastomosis }\end{array}$ & $\begin{array}{l}\text { phiL/phiT, QT, JT } \\
\text { dispersions before, } \\
\text { during and after IP } \\
\text { and during and } \\
\text { after coronary } \\
\text { anastomosis }\end{array}$ & $\begin{array}{l}\text { Anisotropy was exaggerated during the } \\
\text { 5-minute coronary occlusion; during } \\
\text { anastomosis, conduction velocities were } \\
\text { decreased, but showed no further } \\
\text { deterioration; QT and JT dispersions } \\
\text { were improved by reperfusion }\end{array}$ & $\begin{array}{l}\text { Anisotropy and dispersions were } \\
\text { minimized after IP, therefore IP } \\
\text { demonstrated antiarrhythmic } \\
\text { protective effects on the } \\
\text { human myocardium }\end{array}$ \\
\hline \multirow{3}{*}{$\begin{array}{l}\text { Laurikka et al. (2003) Chest, } \\
\text { Finland [14] Prospective } \\
\text { controlled randomized trial }\end{array}$} & $\begin{array}{l}\text { Thirty-two } \\
\text { OPCAB }\end{array}$ & \multirow{3}{*}{$\begin{array}{l}\text { IP induced with cycle of twice } \\
2 \text { min LAD occlusion followed } \\
3 \text { min reperfusion before the } \\
\text { first anastomosis }\end{array}$} & \multirow[t]{3}{*}{$\begin{array}{l}\text { Myocardial } \\
\text { performance }\end{array}$} & \multirow{3}{*}{$\begin{array}{l}\text { IP group had complete recovery of mean } \\
\text { after the operation; in the control } \\
\text { subjects, mean SVI showed a } \\
\text { significant reduction postoperatively }\end{array}$} & \multirow{3}{*}{$\begin{array}{l}\text { IP tended to decrease the immediate } \\
\text { myocardial enzyme release, prohibited } \\
\text { the postoperative increase in HR, and } \\
\text { enhanced the recovery of SVI }\end{array}$} \\
\hline & $16 \mathrm{IP}$ & & & & \\
\hline & 16 Control & & & & \\
\hline \multirow{2}{*}{$\begin{array}{l}\text { Matsumoto et al. (2001) } \\
\text { Kyobu Geka, Japan [24] } \\
\text { Retrospective observational } \\
\text { study }\end{array}$} & $\begin{array}{l}\text { Forty-three } \\
\text { OPCAB }\end{array}$ & \multirow{2}{*}{$\begin{array}{l}\text { IP induced with twice } 5 \text { min } \\
\text { vessel occlusion followed } 5 \text { min } \\
\text { reperfusion before anastomosis } \\
\text { Allopurinol preoperatively and } \\
\text { nicorandil intraoperatively; }\end{array}$} & \multirow[t]{2}{*}{$\begin{array}{l}\text { Myocardial tissue } \\
\text { oxygen saturation }\end{array}$} & \multirow{2}{*}{$\begin{array}{l}\text { Troponin level was statistically significant } \\
\text { lower in the IP groupOn day } 1 \text { post op, } \\
\text { the increase in the mean HR was also } \\
\text { significantly lower in the IP group } \\
\text { Significant amelioration of post-ischemic } \\
\text { recovery in the IP + pharmacological } \\
\text { preconditioning }\end{array}$} & \multirow{2}{*}{$\begin{array}{l}\text { Concomitant use of IP and KATP opener, } \\
\text { oxidative radical scavenger both } \\
\text { ameliorated cardiac dysfunction during } \\
\text { ischemia in anastomotic occlusion of } \\
\text { the coronary artery and improved the } \\
\text { post-ischemic functional recovery }\end{array}$} \\
\hline & $\begin{array}{l}29 \mathrm{IP}+ \\
\text { pharmacological }\end{array}$ & & & & \\
\hline \multirow{2}{*}{$\begin{array}{l}\text { van Aarnhem et al. (1999) } \\
\text { Eur J Cardiothorac Surg, } \\
\text { The Netherlands [12] } \\
\text { Retrospective observational } \\
\text { study }\end{array}$} & \multirow[t]{2}{*}{$\begin{array}{l}\text { Two-hundred } \\
\text { OPCAB }\end{array}$} & \multirow[t]{2}{*}{$\begin{array}{l}\text { IP induced with } 5 \text { min of local } \\
\text { coronary artery occlusion and } \\
5 \text { min of reperfusion before } \\
\text { anastomosis }\end{array}$} & \multirow[t]{2}{*}{$\begin{array}{l}\text { Ischemia during } \\
\text { temporary } \\
\text { coronary artery } \\
\text { occlusions }\end{array}$} & $\begin{array}{l}\text { Ischemia (defined as defined } \\
\text { as }>1 \mathrm{~mm} \text { S-T segment) occurred } \\
\text { during } 35(10 \%) \text { temporary coronary } \\
\text { artery occlusions }\end{array}$ & \multirow{4}{*}{$\begin{array}{l}\text { Temporary segmental occlusion was safe } \\
\text { before anastomosis in OPCAB; shunts } \\
\text { were used in critical ischemialschemic } \\
\text { dysfunction was precipitated by the } \\
5 \text {-min LAD occlusion, as shown by the } \\
\text { increase in LVWMS and PA pressure. } \\
\text { However, a 5-min coronary occlusion } \\
\text { and the resulting ischemia did not alter } \\
\text { regional LV systolic function during } \\
\text { subsequent ischemia }\end{array}$} \\
\hline & & & & \multirow{3}{*}{$\begin{array}{l}\text { There were no perioperative MI/no } \\
\text { conversion to ONCAB LWWMS decreased } \\
\text { significantly after first cycle but improved } \\
\text { after IP No significant differences in } \\
\text { pulmonary artery pressures were } \\
\text { after IP and during anastomosis }\end{array}$} & \\
\hline \multirow{2}{*}{$\begin{array}{l}\text { Malkowski (1998) J Am Coll } \\
\text { Cardiol (USA) [13] Prospective } \\
\text { observational study }\end{array}$} & \multirow{2}{*}{$\begin{array}{l}\text { Seventeen } \\
\text { OPCAB } \\
\text { (MIDCAB) }\end{array}$} & \multirow{2}{*}{$\begin{array}{l}\text { IP induced with } 5 \text { min of } \\
\text { local coronary artery } \\
\text { occlusion and } 5 \text { min } \\
\text { of reperfusion }\end{array}$} & LWMS & & \\
\hline & & & $\begin{array}{l}\text { PA systolic and } \\
\text { diastolic pressure }\end{array}$ & & \\
\hline
\end{tabular}

AF: Atrial fibrillation; IP: Ischemic preconditioning; I/R: Ischemia reperfusion; LAD: Left anterior descending artery; LVWMS: Left ventricle wall motion score; MIDCAB: Minimally invasive direct coronary artery bypass grafting; ONCAB: On-pump CABG; OPCAB: Off-pump CABG; phiL/phiT: Ratio of longitudinal to transverse conduction velocity PostC: Postconditioning; RIPC: Ischemic remote preconditioning; SVI: Stroke volume index; SS: Sevoflurane. 
Table 2 Studies including anaesthetics agents

Mroziński et al. (2014) Sixty OPCAB 28

Anaesthesiol Intensive Ther,

Propofol 32

DES PP

randomized open-label trial

Orriach et al. (2013) J Crit Care, Spain [25] Prospective

randomized trial

Wang et al. (2013)

Scand Cardiovasc J, China

[26] Prospective randomized controlled trial

Suryaprakash et al. (2013)

Ann Card Anaesth, India [28]

Prospective randomized trial

Tempe et al. (2011)

J Cardiothorac Vasc Anesth,

India [38] Prospective

randomized trial

Ballester et al. (2011)

Eur J Anaesthesiol, Spain [33]

Prospective controlled

randomized trial

Kim et al. (2011)

Anaesth Intensive Care, Korea

[29] Prospective randomized

controlled trial

Hammerling et al. (2010)

Ann Card Anaesth Canada [34]

Prospective double blinded trial

BNP Troponin release Need SS group had reduced BNP, troponin

for inotropic drugs release and number of inotropic

drugs Compared to S-P and

P-P groups

BNP

Troponin release

20 Sevoflurane

20 Control

One hundred

thirty

nine $O P C A B$

48 Sevoflurane

PP

52 Desflurane

39 Propofol

Forty-five OPCAB ISO

Isoflurane PP

Propofol

Thirty-eight

OPCAB

20 Sevoflurane

18 Propofol

Ninety-four

OPCAB

47 Sevoflurane

47 Propofol

(both in a

remifentanil based

anesthesia)

Forty OPCAB

20 Sevoflurane

DES

SS significantly decreased post-surgical in BNP level among groups

Changes in troponin levels at all time intervals were comparable in the three groups

Troponin release

Troponin release in the PP group was 6 and 24 hours after surgery

Markers of lipoperoxidation (F2-isoprostanes) and

nitrosative stress

(nitrates/nitrites) measured

in coronary sinus blood

CK MB and troponin release No statistically differences in between release at different end points

Incidence of arrhythmias in the DES-group, AF was significantly

20 Desflurane

Assessment of hemodynamic

DES group demonstrated improved

stability, expressed as LVSWl; no

injury markers

between groups

No difference reported between DES and PP in major haemodynamic

parameters, myocardial injury markers

and the long-term outcome; DES might accelerate LVSWI recovery

SS administration in $\mathrm{OR}$ and $\mathrm{ClCU}$ decreased troponin release compared with SS intra-op, but both were a better option to decrease troponin level when compared to PP troponin levels No significant differences significantly higher than the ISO group at groups in terms of CK-MB and troponin

Supraventricular tachycardia occurred only more frequent in the DES group versus SEVO-group
SS exerted significant myocardial protective effect; BNP could not predict myocardial protective effect of SS in OPCA

No difference found in myocardia protection with SS or DES or PP

ISO provided protection against myocardial damage by lowering levels of troponin-T

SS showed better antioxidative properties than PP

SS and PP had similar CK-MB and troponin values

SS found to be more advantageous than DES, as it was associated with ess AF or supraventricular arrhythmias 
Table 2 Studies including anaesthetics agents (Continued)

\begin{tabular}{|c|c|c|c|c|c|}
\hline \multirow{3}{*}{$\begin{array}{l}\text { Xu (2009) J South Med Univ, } \\
\text { China [42] Prospective } \\
\text { controlled randomized trial }\end{array}$} & $\begin{array}{l}\text { Twenty four } \\
\text { OPCAB }\end{array}$ & \multirow[t]{3}{*}{ REMI } & \multirow[t]{3}{*}{ Troponin release } & \multirow[t]{3}{*}{$\begin{array}{l}\text { Statistically significant reduction of } \\
\text { troponin level in the REMI group }\end{array}$} & \multirow{3}{*}{$\begin{array}{l}\text { Troponin levels of REMI preconditioning } \\
\text { group were markedly decreased after } \\
\text { the operation in comparison with } \\
\text { those of the control group }\end{array}$} \\
\hline & 12 Remifentanil & & & & \\
\hline & 12 Control & & & & \\
\hline \multirow{4}{*}{$\begin{array}{l}\text { Drenger et al. (2008) Journal of } \\
\text { Cardiothoracic and Vascular } \\
\text { Anesthesia, Israel [21] Prospective } \\
\text { controlled randomized trial }\end{array}$} & $\begin{array}{l}\text { Twenty five } \\
\text { OPCAB }\end{array}$ & \multirow{4}{*}{$\begin{array}{l}\text { IP induced with } \\
\text { single } 5 \text { min } L A D \\
\text { occlusion followed } \\
\text { by } 5 \text { min reperfusion } \\
1.6 \% \text { ENF started } \\
15 \text { min before } \\
\text { LAD occlusion }\end{array}$} & \multirow{4}{*}{ Myocardial metabolism } & \multirow{4}{*}{$\begin{array}{l}\text { Lactate production in the ENF } \\
\text { group decreased significantly } \\
\text { compared with control and IP } \\
\text { groups. Oxygen utilization in the } \\
\text { control group was } 44 \% \text { higher than } \\
\text { the other two groups Early recovery of } \\
\text { anterior wall hypokinesis in both } \\
\text { study groups }\end{array}$} & \multirow{4}{*}{$\begin{array}{l}\text { Application of methods such as IP or } \\
\text { volatile anesthesia appeared to } \\
\text { reduce the metabolic deficit }\end{array}$} \\
\hline & 8 Control & & & & \\
\hline & $9 \mathbb{P}$ & & & & \\
\hline & 8 Enflurane & & & & \\
\hline \multirow{3}{*}{$\begin{array}{l}\text { Hemmerling (2008) European Journal } \\
\text { of Anaesthesiology, Canada [31] } \\
\text { Prospective randomised trial }\end{array}$} & Fourty OPCAB & SS & Troponin/CK-MB & \multirow{3}{*}{$\begin{array}{l}\text { No differences in terms of enzymes } \\
\text { release, heart contractility and } \\
\text { haemodynamic values Extubation } \\
\text { time was significantly shorter with } \\
\text { SS compared to ISO }\end{array}$} & \multirow{3}{*}{$\begin{array}{l}\text { SS and ISO provided the same ischaemic } \\
\text { cardio-protective effects; SEVO allowed } \\
\text { a more rapid recovery from anaesthesia }\end{array}$} \\
\hline & 20 Sevoflurane & ISO & LWWM abnormalities time to & & \\
\hline & 20 Isoflurane & & $\begin{array}{l}\text { functions haemodynamic } \\
\text { parameters }\end{array}$ & & \\
\hline \multirow{3}{*}{$\begin{array}{l}\text { Huseidzinović et al. (2007) } \\
\text { Croat Med J, Croatia [35] } \\
\text { Prospective randomised } \\
\text { controlled trial }\end{array}$} & 32 OPCABG & \multirow[t]{3}{*}{ SS } & \multirow{3}{*}{$\begin{array}{l}\text { Acceleration of aortic blood } \\
\text { flow, Cl, HR, mean arterial } \\
\text { pressure, and central venous } \\
\text { pressure at different time } \\
\text { points }\end{array}$} & \multirow{3}{*}{$\begin{array}{l}\text { SS group showed better Cl values at } \\
\text { the beginning of ischemia and } 15 \text { minutes } \\
\text { after ischemia; in the PP group acceleration } \\
\text { decreased and remained lower } 15 \text { minutes } \\
\text { after sternal closure while was increased } \\
\text { in the SS group }\end{array}$} & \multirow{3}{*}{$\begin{array}{l}\text { Cardiac function was better preserved } \\
\text { in patients with SS than with PP }\end{array}$} \\
\hline & 16 Sevoflurane & & & & \\
\hline & 16 Control & & & & \\
\hline \multirow{3}{*}{$\begin{array}{l}\text { Venkatesh et al. (2007) } \\
\text { Ann Card Anaesth, India [32] } \\
\text { Prospective randomized }\end{array}$} & Forty OPCAB & SS & \multirow{3}{*}{$\begin{array}{l}\text { Haemodynamic effects } \\
\text { amount of analgesia needed } \\
\text { postoperative recovery }\end{array}$} & \multirow{3}{*}{$\begin{array}{l}\text { No differences identified in terms of } \\
\text { haemodynamic parameters, depth of } \\
\text { anesthesia, and quantity of agent } \\
\text { needed; time of awakening and } \\
\text { subsequent extubation were } \\
\text { significantly less with SS }\end{array}$} & \multirow{3}{*}{$\begin{array}{l}\text { SS and ISO could both safely used in } \\
\text { OPCAB; awakening and extubation } \\
\text { time were significantly lower with SS. }\end{array}$} \\
\hline & 20 Isoflurane & ISO & & & \\
\hline & 20 Sevoflurne & & & & \\
\hline \multirow{3}{*}{$\begin{array}{l}\text { Lucchinetti et al. (2007) } \\
\text { Anesthesiology, Switzerland [37] } \\
\text { Prospective randomised trial }\end{array}$} & $20 \mathrm{OPCAB}$ & SS & \multirow{3}{*}{$\begin{array}{l}\text { Troponin, NBP and associate } \\
\text { pregnancy-associated plasma } \\
\text { protein A release Gene } \\
\text { expression profile } \\
\text { (atrial biopsies) }\end{array}$} & \multirow{3}{*}{$\begin{array}{l}\text { NPB and protein A were decrease in SS } \\
\text { group; Echo showed preserved post-op } \\
\text { LV function in SS group. }\end{array}$} & \multirow{3}{*}{$\begin{array}{l}\text { SS gene regulatory control of myocardial } \\
\text { substrate metabolism predicted } \\
\text { postoperative cardiac function in } \\
\text { OPCAB patients }\end{array}$} \\
\hline & 10 Propofol & PP & & & \\
\hline & 10 Sevoflurane & & & & \\
\hline \multirow{4}{*}{$\begin{array}{l}\text { Guarracino et al. (2006) Journal of } \\
\text { Cardiothoracic and Vascular } \\
\text { Anesthesia, Italy [41] Prospective } \\
\text { randomised trial }\end{array}$} & $\begin{array}{l}\text { One hundred } \\
\text { twelve }\end{array}$ & DES & \multirow[t]{4}{*}{ Troponin release } & \multirow[t]{4}{*}{$\begin{array}{l}\text { Post-op peak troponin was significantly } \\
\text { lower in DES group }\end{array}$} & \multirow{4}{*}{$\begin{array}{l}\text { Myocardial damage measured by } \\
\text { cardiac troponin release could be } \\
\text { reduced by DES during OPCAB }\end{array}$} \\
\hline & OPCAB & PP & & & \\
\hline & 57 Desflurane & \multirow{2}{*}{$\begin{array}{l}\text { (in addition to } \\
\text { opiate-based } \\
\text { anesthesia) }\end{array}$} & & & \\
\hline & 55 Propofol & & & & \\
\hline
\end{tabular}


Table 2 Studies including anaesthetics agents (Continued)

\begin{tabular}{|c|c|c|c|c|c|}
\hline \multirow{3}{*}{$\begin{array}{l}\text { Law-Koune (2006) J Cardiothorac } \\
\text { Vasc Anesth, France [30] Prospective } \\
\text { randomized trial }\end{array}$} & Eighteen OPCAB & SS-REMI & \multirow[t]{3}{*}{ Troponin release } & \multirow[t]{3}{*}{ No difference in troponin release } & \multirow{3}{*}{$\begin{array}{l}\text { Study did not support cardio-protective } \\
\text { effects of SS }\end{array}$} \\
\hline & $\begin{array}{l}9 \text { Sevoflurane- } \\
\text { remifentanil }\end{array}$ & \multirow[t]{2}{*}{ PP-REMI } & & & \\
\hline & $\begin{array}{l}9 \text { Propofol- } \\
\text { remifentanil }\end{array}$ & & & & \\
\hline \multirow{3}{*}{$\begin{array}{l}\text { Bein et al. (2005) Anesth Analg, } \\
\text { Germany [43] Prospective } \\
\text { randomized trial }\end{array}$} & $\begin{array}{l}\text { Fifty-two OPCAB } \\
\text { (MIDCAB) }\end{array}$ & SS & \multirow[t]{3}{*}{ Myocardial function } & \multirow{3}{*}{$\begin{array}{l}\text { Myocardial performance index and } \\
\text { early to atrial filling velocity ratio in the } \\
\text { PP group deteriorated significantly } \\
\text { whereas there was no change in } \\
\text { the SS group }\end{array}$} & \multirow{3}{*}{$\begin{array}{l}\text { In patients undergoing MIDCAB surgery, } \\
\text { SS preserved myocardial function } \\
\text { better than PP }\end{array}$} \\
\hline & 26 Sevoflurane & \multirow[t]{2}{*}{ PP } & & & \\
\hline & 26 Propofol & & & & \\
\hline \multirow{4}{*}{$\begin{array}{l}\text { Kendall (2004) Anaesthesia, } \\
\text { UK [39] Prospective } \\
\text { randomized trial }\end{array}$} & Thirty OPCAB & PP & \multirow[t]{4}{*}{ Troponin release } & \multirow{4}{*}{$\begin{array}{l}\text { No significant difference in } \\
\text { between groups }\end{array}$} & \multirow{4}{*}{$\begin{array}{l}\text { No support of ISO as cardioprotective } \\
\text { agent was reported }\end{array}$} \\
\hline & 10 Propofol & ISO & & & \\
\hline & 10 Isoflurane & \multirow{2}{*}{$\begin{array}{l}\text { ISO/high thoracic } \\
\text { epidural analgesia }\end{array}$} & & & \\
\hline & $\begin{array}{l}10 \text { Isoflurane and } \\
\text { high thoracic } \\
\text { epidural analgesia }\end{array}$ & & & & \\
\hline \multirow{3}{*}{$\begin{array}{l}\text { Conzen et al. (2003) } \\
\text { Anesthesiology, Germany [27] } \\
\text { Prospective randomized trial }\end{array}$} & Twenty OPCAB & \multirow[t]{3}{*}{ SS } & \multirow[t]{3}{*}{ Troponin release } & \multirow{3}{*}{$\begin{array}{l}\text { Troponin increased significantly more in } \\
\text { the PP group rather than in the SS group }\end{array}$} & \multirow{3}{*}{$\begin{array}{l}\text { Patients receiving SS had less myocardial } \\
\text { injury during the first } 24 \text { post-op hours } \\
\text { than patients with PP }\end{array}$} \\
\hline & 10 Sevoflurane & & & & \\
\hline & 10 Propofol & & & & \\
\hline
\end{tabular}

AF: Atrial fibrillation; BNP: Brain natriuretic peptide ONCAB: On pump Coronary Artery By-pass Grafting; OPCAB: Off pump Coronary Artery By-pass Grafting; DES: Desflurane IP: Ischemic preconditioning; LAD: Left anterior descending artery; LVWMS: Left ventricle wall motion score; MIDCAB: Minimally invasive direct coronary artery bypass grafting; PostC: Postconditioning; SS: Sevoflurane. 
preconditioned groups in terms of EF and troponin release. Li et al. [6] randomized a small number of patients to on and off-pump to be preconditioned with the use of hyperbaric oxygen (HBO). Patients in the preconditioning group underwent $\mathrm{HBO}$ for $70 \mathrm{~min} /$ daily for $5 \mathrm{con}$ secutive days before surgery. Preconditioning with $\mathrm{HBO}$ resulted in both cerebral and cardiac protective effects as determined by biochemical markers of neuronal and myocardial injury and clinical outcomes in patients undergoing $\mathrm{ONCAB}$ while no benefits were observed in the OPCAB group. Matsumoto et al. [24] reviewed a cohort of 48 OPCAB patients. Among them, the subgroup treated with IP plus allopurinol and nicorandil had an improved post-ischemic functional recovery after surgery.

Myocardial conditioning in cardiac surgery can be achieved in different ways. The most used are central or remote preconditioning and volatile anesthetics, while adenosine or other pharmacological agents less frequently used. Ischemic conditioning was first reported in 1986 [4] and conditioning by volatile anesthetic with halothane in 1976 [44]. Although different, both techniques share probably some common mechanisms of action and, most importantly, can be used simultaneously. Both have been frequently utilized in ONCAB [45], but with regard of OPCAB surgery no large trials have been published so far evaluating their effect on troponin [19] or clinical outcomes. Moreover, according to clinical trial.gov [46], while there are different trials in different phases, registered and ongoing, investigating the effect of RIPC in ONCABG (isolated or plus/minus valve surgery) the only trial aiming to compare the effect of RIPC on ON and OFF-pump CABG is the RIP-CON study, expected to report in June 2015 [47]. Small trials are present in the literature, focusing on the effect of RIPC on different subsystem outcomes. Joung et al. [16] randomised 70 OPCAB patients to RIPC and control with cognitive function as primary end points. Hong et al. reported no differences in terms of troponin release in OPCAB after RIPC [19]. Same Authors [18], however, studied the effect of RIPC and PostC together and they observed, this time, a reduction of troponin release in a series of OPCAB. All the other trials [13,14,17,20-22,48] carried out in $\mathrm{OPCAB}$ with conditioning used 'central preconditioning, hence eliciting protection by cycles of occlusion/reperfusion at the level of targeted vessel. All of them led to different and non-homogeneous results. Central remote ischemic preconditioning has been, however, fairly dismissed in on pump cardiac surgery and during interventional cardiology procedure for its impracticality and its high level of invasiveness $[2,3,49]$. It is important to stress that some of the RIPC and central IP trials did not state the type of anesthesia used [13,23], or deliberately used propofol as anesthetic maintenance $[12,16-18,20]$, while very few did not use it $[19,21,22]$.
Recently it has been reported that propofol may inhibit preconditioning effect, and large trials have intentionally avoided it use [50].

Since the first report in 1976 [44] volatile anaesthetics have been frequently used as conditioning techniques in ONCAB. Two large meta-analyses from 2006 [51,52] have suggested that there was a significant difference in in patients who received volatile agents in terms of reduced troponin release, improved CI, less use of inotropes and reduced need of mechanical ventilation. However they did not point out any differences with regards of mortality, LOS and myocardial infarction rate. Moreover a relatively recent review [53] has reported that sevoflurane had beneficial effects only in naïve patients with no previous exposures to episode of angina. Although there is sufficient evidence to support the use of volatile agents during cardiac surgery and taking into account their feasibility to be used as pre, per and post conditioning with the potential to prolong the protective effect [25], no large trials have been set in place in order to identify potential benefits in patients undergoing $\mathrm{OPCAB}$. Up till now there are 17 trials comparing different volatile anesthetics $v s$ propofol or $v s$ control or volatiles among themself but the vast majority of them were underpowered and led to conflictive results (Table 2). Sevoflurane is proven to reduce troponin level after on-pump surgery but its effect is still unclear in the off-pump scenario. With regard to OPCAB, sevoflurane was found to preserve cardiac function in three studies $[35,37,54]$, to reduce troponin release in two studies $[26,27]$, to have antioxidant properties in one [33] and to prevent incidence of arrhythmias in one [34]. On the contrary sevoflurane did not show any better troponin reduction when compared to other agents in four studies [28-31]. Only Orriach et al. used sevoflurane during and after the operation as post-conditioning and reported a significant reduction in troponin release [25].

\section{Conclusions}

Although $\mathrm{OPCAB}$ is thought to reduce the extent of general ischemia, ischemic cardiac insult can be found in up to $10 \%$ of the patients [12]. Data coming from the largest pooled analysis on mainly on-pump experience reported a trend toward a consistent reduction of almost a half of MI in RIPC groups [55]; hence there may be the theoretical potential to translate the same advantage to off-pump patients. However, up till now there are no strong evidences supporting the use of ischemic preconditioning, either central or remote, in OPCAB patients.

In terms of volatile anaesthetics, taking into account all the trials, we can conclude here that has not yet been demonstrated if they can reduce troponin level after off-pump surgery. Other forms of conditioning used in $\mathrm{OPCAB}$ such as adenosine/HBO/pharmacological have been used and some times in association with IP, but led 
to different conclusions. Conditioning, either elicited by ischemia/reperfusion or by volatile agents, may theoretically be a valid method to increase cardiac protection in off-pump surgery, but further trials are definitely needed.

\author{
Abbreviations \\ AF: Atrial fibrillation; BNP: Brain natriuretic peptide; CABG: Coronary Artery \\ By-pass Grafting; DES: Desflurane; HBO: Hyperbaric oxygen; IP: Ischemic \\ preconditioning; I/R: Ischemia reperfusion; LAD: Left anterior descending \\ artery; LWWMS: Left ventricle wall motion score; MIDCAB: Minimally invasive \\ direct coronary artery bypass grafting; ONCAB: On-pump CABG; \\ OPCAB: Off-pump CABG; phiL /phiT: Ratio of longitudinal to transverse \\ conduction velocity; PostC: Postconditioning; RIPC: Ischemic remote \\ preconditioning; SVI: Stroke volume index; SS: Sevoflurane; TIVA: Total \\ intra-venous anesthesia.
}

\section{Competing interests}

The authors declare that they have no competing interests.

\section{Authors' contributions}

MM, PPP reviewed literature, collected data and wrote the manuscript. PDS critically reviewed anaesthetic literature. GM, GDA, FF revised and critically reviewed the manuscript. All authors read and approved the final manuscript.

\section{Author details}

${ }^{1} \mathrm{NHLI}$, Hammersmith Hospital, Imperial College London, London, UK. ${ }^{2}$ Fondazione Monasterio, Ospedale Pasquinucci, Massa, Italy. ${ }^{3}$ Bristol Heart Institute, University of Bristol, Bristol, UK.

Received: 20 October 2014 Accepted: 26 December 2014 Published online: 20 January 2015

\section{References}

1. Kharbanda RK. Cardiac conditioning: a review of evolving strategies to reduce ischaemia-reperfusion injury. Heart. 2010;96:1179-86.

2. Hausenloy DJ, Mwamure PK, Venugopal V, Harris J, Barnard M, Grundy E, et al. Effect of remote ischaemic preconditioning on myocardial injury in patients undergoing coronary artery bypass graft surgery: a randomised controlled trial. Lancet. 2007:370:575-9.

3. Jenkins DP, Pugsley WB, Alkhulaifi AM, Kemp M, Hooper J, Yellon DM. Ischaemic preconditioning reduces troponin t release in patients undergoing coronary artery bypass surgery. Heart. 1997;77:314-8.

4. Murry CE, Jennings RB, Reimer KA. Preconditioning with ischemia: a delay of lethal cell injury in ischemic myocardium. Circulation. 1986;74:1124-36.

5. Jones WK, Fan GC, Liao S, Zhang JM, Wang Y, Weintraub NL, et al. Peripheral nociception associated with surgical incision elicits remote nonischemic cardioprotection via neurogenic activation of protein kinase $c$ signaling. Circulation. 2009;120:S1-9.

6. Li Y, Dong H, Chen M, Liu J, Yang L, Chen S, et al. Preconditioning with repeated hyperbaric oxygen induces myocardial and cerebral protection in patients undergoing coronary artery bypass graft surgery: a prospective, randomized, controlled clinical trial. J Cardiothorac Vasc Anesth. 2011;25:908-16.

7. Zaugg M, Lucchinetti E, Spahn DR, Pasch T, Schaub MC. Volatile anesthetics mimic cardiac preconditioning by priming the activation of mitochondrial k(atp) channels via multiple signaling pathways. Anesthesiology. 2002;97:4-14.

8. Thielmann M, Kottenberg E, Kleinbongard P, Wendt D, Gedik N, Pasa S, et al. Cardioprotective and prognostic effects of remote ischaemic preconditioning in patients undergoing coronary artery bypass surgery: a single-centre randomised, double-blind, controlled trial. Lancet. 2013;382:597-604.

9. Xie JJ, Liao XL, Chen WG, Huang DD, Chang FJ, Chen W, et al. Remote ischaemic preconditioning reduces myocardial injury in patients undergoing heart valve surgery: randomised controlled trial. Heart. 2012;98:384-8

10. Meybohm P, Zacharowski K, Cremer J, Roesner J, Kletzin F, Schaelte G, et al. Remote ischaemic preconditioning for heart surgery. The study design for a multi-center randomized double-blinded controlled clinical trial-the ripheart-study. Eur Heart J. 2012;33:1423-6.

11. Hausenloy DJ, Candilio L, Laing C, Kunst G, Pepper J, Kolvekar S, et al. Effect of remote ischemic preconditioning on clinical outcomes in patients undergoing coronary artery bypass graft surgery (ericca): rationale and study design of a multi-centre randomized double-blinded controlled clinical trial. Clin Res Cardiol Off J German Cardiac Soc. 2012;101:339-48.

12. van Aarnhem EE, Nierich AP, Jansen EW. When and how to shunt the coronary circulation in off-pump coronary artery bypass grafting. Eur J Cardio-Thoracic Surgery Off J Eur Assoc Cardio-Thoracic Surgery. 1999;16 Suppl 2:S2-6.

13. Malkowski MJ, Kramer CM, Parvizi ST, Dianzumba S, Marquez J, Reichek N, et al. Transient ischemia does not limit subsequent ischemic regional dysfunction in humans: a transesophageal echocardiographic study during minimally invasive coronary artery bypass surgery. J Am Coll Cardiol. 1998;31:1035-9.

14. Laurikka J, Wu ZK, lisalo P, Kaukinen L, Honkonen EL, Kaukinen S, et al. Regional ischemic preconditioning enhances myocardial performance in off-pump coronary artery bypass grafting. Chest. 2002;121:1183-9.

15. Chowdhury R, White D, Kilgo P, Puskas JD, Thourani VH, Chen EP, et al. Risk factors for conversion to cardiopulmonary bypass during off-pump coronary artery bypass surgery. Ann Thorac Surg. 2012;93:1936-41. discussion 1942.

16. Joung KW, Rhim JH, Chin JH, Kim WJ, Choi DK, Lee EH, et al. Effect of remote ischemic preconditioning on cognitive function after off-pump coronary artery bypass graft: a pilot study. Korean J Anesthesiol. 2013;65:418-24

17. Forouzannia SK, Abdollahi MH, Mirhosseini SJ, Hadadzadeh M, Zarepur R, Zarepur $\mathrm{E}$, et al. Adenosine preconditioning versus ischemic preconditioning in patients undergoing off-pump coronary artery bypass (opcab). J Tehran Heart Center. 2013;8:127-31.

18. Hong DM, Jeon Y, Lee CS, Kim HJ, Lee JM, Bahk JH, et al. Effects of remote ischemic preconditioning with postconditioning in patients undergoing off-pump coronary artery bypass surgery-randomized controlled trial. Circulation J Off J Jpn Circulation Soc. 2012;76:884-90.

19. Hong DM, Mint JJ, Kim JH, Sohn IS, Lim TW, Lim YJ, et al. The effect of remote ischaemic preconditioning on myocardial injury in patients undergoing off-pump coronary artery bypass graft surgery. Anaesth Intensive Care. 2010;38:924-9.

20. Succi JE, Gerola LR, Succi GM, Almeida RA, Novais LS, Rocha B. Ischemic preconditioning influence ventricular function in off-pump revascularization surgery. Arq Bras Cardiol. 2010;94:319-24.

21. Drenger B, Gilon D, Chevion M, Elami A, Meroz Y, Milgalter E, et al. Myocardial metabolism altered by ischemic preconditioning and enflurane in off-pump coronary artery surgery. J Cardiothorac Vasc Anesth. 2008:22:369-76.

22. Wu ZK, livainen T, Pehkonen E, Laurikka J, Tarkka MR. Arrhythmias in off-pump coronary artery bypass grafting and the antiarrhythmic effect of regional ischemic preconditioning. J Cardiothorac Vasc Anesth. 2003;17:459-64.

23. Doi Y, Watanabe G, Kotoh K, Ueyama K, Misaki T. Myocardial ischemic preconditioning during minimally invasive direct coronary artery bypass grafting attenuates ischemia-induced electrophysiological changes in human ventricle. Jpn J Thoracic Cardiovasc Off Publ Japan Assoc Thoracic Surgery = Nihon Kyobu Geka Gakkai zasshi. 2003;51:144-50.

24. Matsumoto $Y$, Endo M, Abe Y, Kasashima F, Kawashima S, Hirano Y, et al. The role of ischemic preconditioning in off-pump cabg: is it really necessary to accomplish scrupulous ischemic preconditioning? Kyobu geka Jpn J Thoracic Surgery. 2001;54:326-31.

25. Guerrero Orriach JL, Galan Ortega M, Ramirez Aliaga M, Iglesias P, Rubio Navarro M, Cruz Manas J. Prolonged sevoflurane administration in the off-pump coronary artery bypass graft surgery: beneficial effects. J Crit Care. 2013;28:879-13. 878.

26. Wang J, Zheng H, Chen CL, Lu W, Zhang YQ. Sevoflurane at 1 mac provides optimal myocardial protection during off-pump cabg. Scand Cardiovasc J SCJ. 2013;47:175-84.

27. Conzen PF, Fischer S, Detter C, Peter K. Sevoflurane provides greater protection of the myocardium than propofol in patients undergoing off-pump coronary artery bypass surgery. Anesthesiology. 2003;99:826-33.

28. Suryaprakash S, Chakravarthy M, Muniraju G, Pandey S, Mitra S, Shivalingappa $B$, et al. Myocardial protection during off pump coronary artery bypass surgery: a comparison of inhalational anesthesia with 
sevoflurane or desflurane and total intravenous anesthesia. Ann Card Anaesth. 2013;16:4-8.

29. Kim TY, Kim DK, Yoon TG, Lim JA, Woo NS, Chee HK, et al. Myocardial injury in remifentanil-based anaesthesia for off-pump coronary artery bypass surgery: an equipotent dose of sevoflurane versus propofol. Anaesth Intensive Care. 2011:39:418-25.

30. Law-Koune JD, Raynaud C, Liu N, Dubois C, Romano M, Fischler M. Sevoflurane-remifentanil versus propofol-remifentanil anesthesia at a similar bispectral level for off-pump coronary artery surgery: no evidence of reduced myocardial ischemia. J Cardiothorac Vasc Anesth. 2006;20:484-92.

31. Hemmerling T, Olivier JF, Le N, Prieto I, Bracco D. Myocardial protection by isoflurane vs. Sevoflurane in ultra-fast-track anaesthesia for off-pump aortocoronary bypass grafting. Eur J Anaesthesiol. 2008;25:230-6.

32. Venkatesh BG, Mehta Y, Kumar A, Trehan N. Comparison of sevoflurane and isoflurane in opcab surgery. Ann Card Anaesth. 2007;10:46-50.

33. Ballester M, Llorens J, Garcia-de-la-Asuncion J, Perez-Griera J, Tebar E, MartinezLeon J, et al. Myocardial oxidative stress protection by sevoflurane vs. Propofol: a randomised controlled study in patients undergoing off-pump coronary artery bypass graft surgery. Eur J Anaesthesiol. 2011;28:874-81.

34. Hemmerling TM, Minardi C, Zaouter C, Noiseux N, Prieto I. Sevoflurane causes less arrhythmias than desflurane after off-pump coronary artery bypass grafting: a pilot study. Ann Card Anaesth. 2010;13:116-22.

35. Huseidzinovic I, Barisin S, Bradic N, Milanovic R. Early cardioprotective effect of sevoflurane on left ventricular performance during coronary artery bypass grafting on a beating heart: randomized controlled study. Croatian Med J. 2007:48:333-40

36. Bein B, Turowski P, Renner J, Hanss R, Steinfath M, Scholz J, et al. Comparison of xenon-based anaesthesia compared with total intravenous anaesthesia in high risk surgical patients. Anaesthesia. 2005;60:960-7.

37. Lucchinetti E, Hofer C, Bestmann L, Hersberger M, Feng J, Zhu M, et al. Gene regulatory control of myocardial energy metabolism predicts postoperative cardiac function in patients undergoing off-pump coronary artery bypass graft surgery: inhalational versus intravenous anesthetics. Anesthesiology. 2007;106:444-57.

38. Tempe DK, Dutta D, Garg M, Minhas H, Tomar A, Virmani S. Myocardial protection with isoflurane during off-pump coronary artery bypass grafting: a randomized trial. J Cardiothorac Vasc Anesth. 2011;25:59-65.

39. Kendall JB, Russell GN, Scawn ND, Akrofi M, Cowan CM, Fox MA. A prospective, randomised, single-blind pilot study to determine the effect of anaesthetic technique on troponin $\mathrm{t}$ release after off-pump coronary artery surgery. Anaesthesia. 2004;59:545-9.

40. Mrozinski P, Lango R, Biedrzycka A, Kowalik MM, Pawlaczyk R, Rogowski J. Comparison of haemodynamics and myocardial injury markers under desflurane vs. Propofol anaesthesia for off-pump coronary surgery. A prospective randomised trial. Anaesthesiol Intensive Therapy. 2014;46:4-13.

41. Guarracino F, Landoni G, Tritapepe L, Pompei F, Leoni A, Aletti G, et al. Myocardial damage prevented by volatile anesthetics: a multicenter randomized controlled study. J Cardiothorac Vasc Anesth. 2006;20:477-83.

42. Xu ZD, Jin M, He WX, Xia SX, Zhao YF, He B, et al. Remifentanil preconditioning lowers cardiac troponin i levels in patients undergoing off-pump coronary artery bypass graft surgery. Nan fang yi ke da xue xue bao $=$ J Southern Med Univ. 2009;29:1554-6.

43. Bein B, Renner J, Caliebe D, Scholz J, Paris A, Fraund S, et al. Sevoflurane but not propofol preserves myocardial function during minimally invasive direct coronary artery bypass surgery. Anesth Analg. 2005;100:610-6. table of contents.

44. Bland $\mathrm{JH}$, Lowenstein E. Halothane-induced decrease in experimental myocardial ischemia in the non-failing canine heart. Anesthesiology. 1976:45:287-93.

45. Pagel PS. Myocardial protection by volatile anesthetics in patients undergoing cardiac surgery: a critical review of the laboratory and clinical evidence. J Cardiothorac Vasc Anesth. 2013;27:972-82.

46. Clinicaltrials.gov www.clinicaltrials.gov/ct2/home.

47. RIPCON trial. www.clinicaltrials.gov/ct2/show/NCT01608984?term=off+pump +preconditioning\&rank=1.

48. Lineker S, Cibere J, Crooks VA, Jones CA, Kopec JA, Lear SA, et al. Capitalizing on the teachable moment: osteoarthritis physical activity and exercise net for improving physical activity in early knee osteoarthritis. J Med Internet Res. 2013;15:1-1.

49. Yellon DM, Alkhulaifi AM, Pugsley WB. Preconditioning the human myocardium. Lancet. 1993;342:276-7.
50. Kottenberg E, Musiolik J, Thielmann M, Jakob H, Peters J, Heusch G. Interference of propofol with signal transducer and activator of transcription 5 activation and cardioprotection by remote ischemic preconditioning during coronary artery bypass grafting. J Thorac Cardiovasc Surg. 2014;147:376-82.

51. Symons JA, Myles PS. Myocardial protection with volatile anaesthetic agents during coronary artery bypass surgery: a meta-analysis. Br J Anaesth. 2006:97:127-36.

52. Yu CH, Beattie WS. The effects of volatile anesthetics on cardiac ischemic complications and mortality in cabg: a meta-analysis. Can J Anaesthesia $=J$ Can d'Anesthesie. 2006:53:906-18

53. Jakobsen CJ, Berg H, Hindsholm KB, Faddy N, Sloth E. The influence of propofol versus sevoflurane anesthesia on outcome in 10,535 cardiac surgical procedures. J Cardiothorac Vasc Anesth. 2007;21:664-71.

54. Bein B, Renner J, Tonner PH. Transoesophageal echocardiography for the determination of cardiac output: beware of improper comparisons. Anaesthesia. 2005;60:512-4.

55. Remote Preconditioning Trialists G, Healy DA, Khan WA, Wong CS, Moloney $M C$, Grace PA, et al. Remote preconditioning and major clinical complications following adult cardiovascular surgery: systematic review and meta-analysis. Int J Cardiol. 2014;176:20-31.

\section{Submit your next manuscript to BioMed Central and take full advantage of:}

- Convenient online submission

- Thorough peer review

- No space constraints or color figure charges

- Immediate publication on acceptance

- Inclusion in PubMed, CAS, Scopus and Google Scholar

- Research which is freely available for redistribution 